\title{
El lenguaje de la insuficiencia cardiaca con fracción de eyección preservada en los últimos treinta años: un análisis bibliométrico
}

\section{The language of heart failure with ejection fraction preserved in the last thirty years: a bibliometric analysis}

\author{
Andrea J. Hernández ${ }^{1 *}$, Carolina Plata ${ }^{1}$, Jacobo Bustamante², Juan E. Múnera², David R. Murillo², \\ Daniela J. Becerra ${ }^{1}$, María Vargas ${ }^{1}$, Edwin F. Mejía ${ }^{1}$, Clara I. Saldarriaga ${ }^{3,4,5}$, Osmar A. Pérez ${ }^{6}$, \\ Adalberto E. Quintero ${ }^{7}$ y Nick Tarazona ${ }^{7}$
}

${ }^{1}$ Departamento de Medicina, Universidad de Santander, Bucaramanga; ${ }^{2}$ Departamento de Medicina, Universidad Tecnológica de Pereira, Pereira; ${ }^{3}$ Departamento de Medicina, Universidad de Antioquia, Medellín; ${ }^{4}$ Departamento de Medicina, Universidad Pontificia Bolivariana, Medellín; ${ }^{5}$ Servicio de Cardiología, Clínica CardioVID, Medellín; ${ }^{6}$ Servicio de Cardiología, Fundación Clínica Shaio, Bogotá; ${ }^{7}$ Departamento de Cardiología del Adulto, Sociedad Colombiana de Cardiología y Cirugía Cardiovascular, Bogotá. Colombia

\section{Resumen}

Introducción: Los estudios bibliométricos permiten realizar un análisis cuantitativo y cualitativo de la producción científica en un campo determinado. En el área de la cardiología, la producción científica ha crecido sustancialmente durante las últimas décadas; sin embargo, existe poca información acerca del manejo integral de la insuficiencia cardiaca con fracción de eyección preservada (ICFEp). Objetivo: Realizar un análisis bibliométrico que proporcione una evaluación detallada del nivel de producción científica de la ICFEp. Método: Estudio bibliométrico descriptivo en el que se realizó una búsqueda en la literatura científica en los últimos 30 años respecto a la ICFEp, para lo cual se utilizó la estrategia de búsqueda "heart failure" AND "preserved ejection fraction" entre 1988 y 2018 en las bases de datos Web of Science, Scopus y Medline a partir de FABUMED y PubReMiner. Resultados: Se encontraron 2830, 4136 y 7943 publicaciones en las bases de datos Medline, Scopus y Web of Science, respectivamente. Los países destacados por su volumen de publicación fueron los Estados Unidos, el Reino Unido y Alemania. En Latinoamérica, los países más productivos fueron Brasil, Argentina y Chile, mientras que Colombia solo tuvo una publicación. Conclusiones: Este análisis bibliométrico es pionero en señalar la evolución de la investigación científica de la ICFEp en los últimos 30 años. Los resultados obtenidos representan un incentivo a la comunidad científica para priorizar la investigación sobre la ICFEp con el fin de mejorar el abordaje integral de los pacientes y, con ello, su calidad de vida.

Palabras clave: Insuficiencia cardiaca. Enfermedad cardiovascular. Análisis bibliométrico.

\section{Abstract}

Introduction: Bibliometric studies are defined as the quantitative and qualitative analysis of scientific production in each field. The scientific production in the area of cardiology has had a substantial growth during the last decades. Despite this, there

\section{Correspondencia:}

*Andrea J. Hernández

E-mail: andreajuliana03@gmail.com
Disponible en internet: 29-11-2021 Rev Colomb Cardiol. 2021;28(5):431-437 www.rccardiologia.com 0120-5633 / @ 2020 Sociedad Colombiana de Cardiología y Cirugía Cardiovascular. Publicado por Permanyer. Este es un artículo open access bajo la licencia CC BY-NC-ND (http://creativecommons.org/licenses/by-nc-nd/4.0/). 
is currently little information about heart failure with preserved ejection fraction (HFpEF). Objective: To provide a detailed assessment of the current status and level of scientific production of HFpEF over the past 30 years. Method: Bibliometric study based in scientific literature concerning HFpEF for the period of 1988 - 2018 using the terms "heart failure" AND "preserved ejection fraction" in the databases of the Web of Science, Scopus and Medline using FABUMED and PubReMiner. Results: We found 2830, 4136 and 7943 publications in Medline, Scopus and Web of Science databases, respectively. The countries with higher scientific production in general were: United States, United Kingdom and Germany. In Latin America, the most productive countries were Brazil, Argentina and Chile. Colombia only had one publication. Conclusions: This is the first bibliometric analysis to highlight the production, evolution and current situation of scientific research about the HFpEF in the last 30 years, a call is made to the scientific community worldwide and especially in our country, Colombia, to focus in the scientific production on this subject. The above mentioned, in order to clarify the pathophysiology, reduce morbidity, mortality and improve the quality of life of patients suffering from HFpEF.

Key words: Heart failure. Cardiovascular disease. Bibliometric analyses.

\section{Introducción}

Los estudios bibliométricos se definen como el análisis cuantitativo y cualitativo de un conjunto de publicaciones sobre diferentes campos temáticos, que son útiles para determinar las tendencias de productividad científica y la orientación de futuras investigaciones ${ }^{1}$. Este tipo de estudios se están realizando con mayor frecuencia debido a la disposición de bases de datos especializadas que facilitan la adquisición de información relevante ${ }^{2}$. Sin embargo, enfermedades como la falla cardiaca carecen de ese tipo de publicaciones en el ámbito nacional, que buscan mejorar las políticas de manejo actual y el pronóstico de los pacientes.

El comportamiento epidemiológico de la insuficiencia cardiaca ha sido variable a lo largo del tiempo. En el mundo afecta a cerca de 40 millones de personas, de las cuales un $2.7 \%$ corresponden a la población colombiana, y de ellos el $60 \%$ son hombres y el $40 \%$ son mujeres ${ }^{3}$. Esta enfermedad se considera un problema de salud pública debido a los elevados costos del tratamiento y los continuos reingresos hospitalarios, como consecuencia del mal control de los factores de riesgo y la diversidad de las subpoblaciones que dificultan su manejo e impactan en la calidad de vida de quienes la padecen ${ }^{2-4}$.

La producción científica en el área de la cardiología ha tenido un crecimiento sustancial durante las últimas décadas. Entre las enfermedades más representativas en este campo se encuentra la insuficiencia cardiaca, un síndrome clínico caracterizado por síntomas típicos (como disnea, edema y fatiga), que puede ir acompañado de signos (presión venosa yugular elevada, crépitos pulmonares y edema periférico), causados por una anomalía cardiaca estructural o funcional, que producen una reducción del gasto cardiaco o una elevación de las presiones intracardiacas en reposo o en estrés ${ }^{5}$.
La insuficiencia cardiaca se clasifica según la fracción de eyección del ventrículo izquierdo, la cual se reporta por ecocardiografía. Se trata de insuficiencia cardiaca con fracción de eyección reducida (ICFEr) cuando es $<50 \%$, en rango medio cuando oscila entre el $40 \%$ y el $49 \%$, y con fracción de eyección preservada (ICFEp) cuando es $>50 \%{ }^{5-7}$. Esta última variante se caracteriza por comprometer la mitad de los casos globales de falla cardiaca ${ }^{8}$. Entre ICFEr y ICFEp se ha encontrado que no existe una diferencia significativa en términos de carga de la enfermedad y costos de atención médica ${ }^{8}$. Por lo tanto, ambos subtipos son igual de importantes y requieren la misma atención por parte de la comunidad científica, así como de los profesionales de la salud.

En la actualidad existe información insuficiente acerca de la ICFEp; las actuales guías clínicas ofrecen pocas recomendaciones de tratamiento basadas en la evidencia para esta variante ${ }^{5,9,10}$. Además, está la limitante de que la mayoría de los resultados de los estudios realizados han sido negativos en el intento de impactar tanto la reducción de las hospitalizaciones como la mortalidad; situación opuesta para la ICFEr, en la que intervenciones con inhibidores de la enzima convertidora de la angiotensina, antagonistas de los receptores de la angiotensina II, inhibición dual de la neprilisina y del receptor de la angiotensina, betabloqueadores y antagonistas del receptor de la aldosterona han demostrado una reducción en la tasa global de desenlaces adversos, evidenciado en los estudios aleatorizados sobre terapias que mejoran el pronóstico de los pacientes con ICFEr, sin beneficio equivalente para la ICFEp ${ }^{11}$.

Lo anterior obliga a replantear el enfoque en investigación científica respecto de la ICFEp en la búsqueda de una mejor comprensión de la morbilidad, la mortalidad y el grado de reversibilidad de esta enfermedad. 


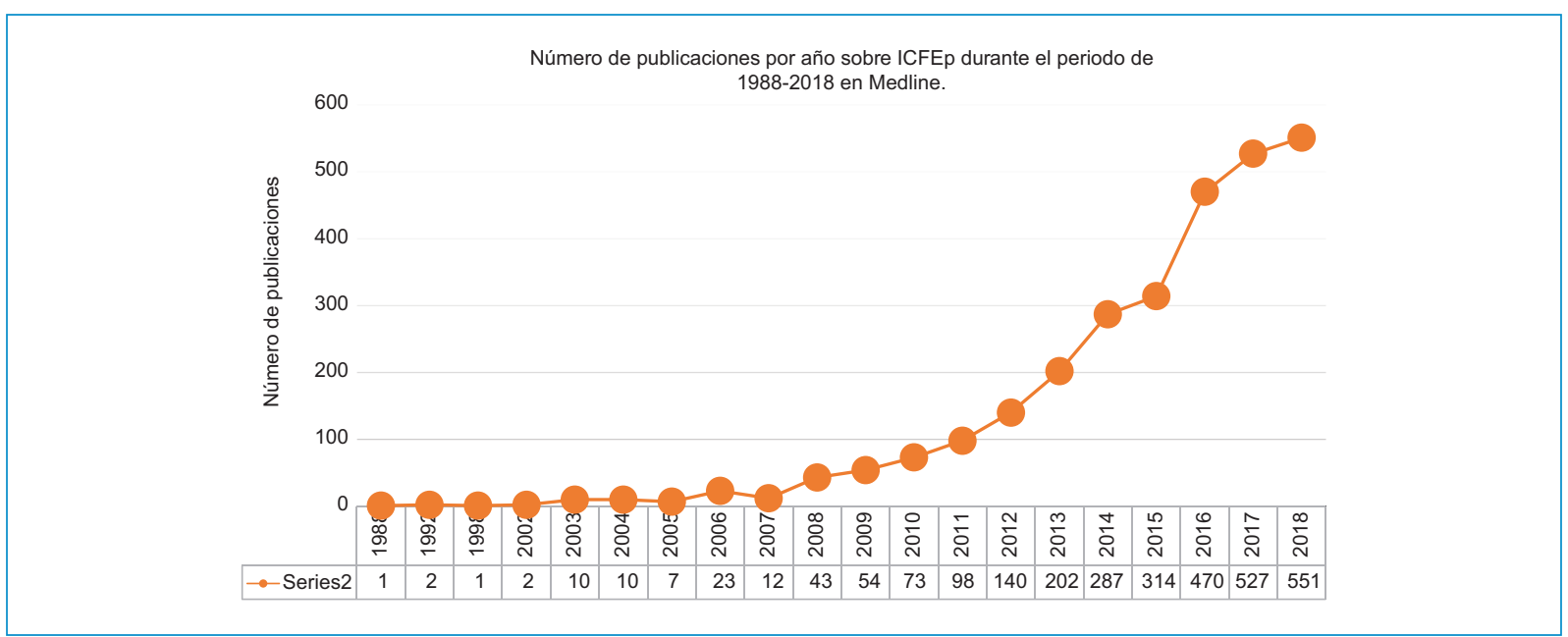

Figura 1. Número de publicaciones por año sobre insuficiencia cardiaca con fracción de eyección preservada durante el periodo 1988-2018, en MEDLINE.

Para ello, en este análisis bibliométrico se proporciona una evaluación detallada del estado actual y del nivel de producción científica sobre la ICFEp en los últimos 30 años.

\section{Método}

Estudio bibliométrico descriptivo en el que se realizó una búsqueda en la literatura científica reportada en los últimos 30 años acerca de la ICFEp. La búsqueda se realizó entre el 1 y el 31 de enero de 2019 utilizando la estrategia "heart failure" AND "preserved ejection fraction" en el periodo de 1988 a 2018, en las bases de datos Web of Science (WoS), Scopus y MEDLINE, a partir de FABUMED y PubReMiner.

Todos los artículos publicados se consideraron para la extracción de información sobre el año de publicación, el tipo de publicación, los países, los autores y las citas, con el fin de obtener una perspectiva generalizada de la capacidad productiva mundial y regional sobre la ICFEp. Estos datos se resumieron desde 1988 hasta 2018 utilizando frecuencias y proporciones.

Se utilizó Microsoft Excel囚 2016 para recopilar la información recolectada, así como para la elaboración de gráficos y tablas de los resultados. Adicionalmente, para evaluar la calidad y la relevancia de las revistas incluidas se accedió a Journal Citation Reports en el periodo 2017-2018, con el fin de determinar el factor de impacto de cada una de ellas, paralelo al rendimiento de los países con las que se encuentran relacionadas.

\section{Resultados}

Del periodo comprendido entre 1988 y 2018 se recolectaron en total 2830 artículos de Medline, en los que se evidenció un incremento importante en la publicación anual, con una tasa de crecimiento del $17.36 \%$. Las revistas más representativas fueron European Journal of Heart Failure, International Journal of Cardiology y Journal of the American College of Cardiology, con una cantidad de artículos publicados de $193(6.81 \%), 124$ (4.38\%) y 116 (4.09\%), respectivamente. Así mismo, en la tabla 1 se identifican aquellas revistas que, durante el periodo 1988-2018, ocuparon los primeros 20 lugares, teniendo en cuenta la cantidad de publicaciones y el factor impacto, al igual que el idioma y el país de procedencia, según el Journal Citation Report de 2017-2018.

Así mismo, se determinaron 78 países con participación científica de acuerdo con las publicaciones que los representan (Figs. 1-2), de los cuales los Estados Unidos ocupó el primer puesto por tener la mayor cantidad de referencias, al contar con un total de 644 publicaciones (22.75\%), seguido del Reino Unido con 335 (11.84\%) y de Alemania con 203 (7.17\%). Por otro lado, entre los países latinoamericanos, el más destacado fue Brasil con 23 referencias $(0.81 \%)$, seguido de México con $10(0.35 \%)$ y Chile con $9(0.31 \%)$; en contraste, Colombia solo cuenta con una referencia bibliográfica sobre el tema $(0.03 \%)$.

En cuanto a los autores de referencia en el tema de la falla cardiaca, de los 8741 autores reportados por Medline, los más destacados, según el volumen de 
Tabla 1. Lista de revistas con artículos publicados sobre insuficiencia cardiaca con fracción de eyección preservada en el periodo 1987-2018, con sus respectivos números de publicaciones, factor de impacto, idioma y país de publicación

\begin{tabular}{|l|c|c|l|l|}
\hline Revista & Artículos & Factor de impacto & Idioma & País \\
\hline European Journal of Heart Failure & 193 & 6.968 & Inglés & Países bajos \\
\hline International Journal of Cardiology & 124 & 6.189 & Inglés & Irlanda \\
\hline Journal of the American College of Cardiology & 116 & 3.398 & Inglés & Estados Unidos \\
\hline Circulation: Heart Failure & 113 & 6.372 & Inglés & Estados Unidos \\
\hline JACC Heart Failure & 104 & 8.493 & Inglés & Estados Unidos \\
\hline Circulation & 97 & 19.309 & Inglés & Estados Unidos \\
\hline Journal of Cardiac Failure & 92 & 3.765 & Inglés & Estados Unidos \\
\hline American Journal of Cardiology & 75 & 19.896 & Inglés & Estados Unidos \\
\hline European Heart Journal & 57 & 19.651 & Inglés & Inglaterra \\
\hline Journal of the American Heart Association & 50 & 9.057 & Inglés & Estados Unidos \\
\hline
\end{tabular}

Tabla 2. Lista de los 10 principales autores con publicaciones de artículos sobre insuficiencia cardiaca con fracción de eyección preservada en el periodo 1897-2018, con sus respectivos números de artículos, ciudad y país de residencia, según MEDLINE

\begin{tabular}{|l|c|l|}
\hline Autor & Publicaciones & Ciudad y país \\
\hline 1. Shah SJ & 105 & Chicago, Estados Unidos \\
\hline 2. Borlaug BA & 99 & Rochester, Estados Unidos \\
\hline 3. Redfield MM & 78 & Rochester, Estados Unidos \\
\hline 4. Solomon SD & 76 & Boston, Estados Unidos \\
\hline 5. Kitzman DW & 66 & $\begin{array}{l}\text { Winston-Salem, Estados } \\
\text { Unidos }\end{array}$ \\
\hline $\begin{array}{l}\text { 6. Pieske B } \\
\text { 7. Zile MR }\end{array}$ & 62 & Berlín, Alemania \\
\hline 8. Buttler J & 58 & Charleston, Estados Unidos \\
\hline $\begin{array}{l}\text { 9. Lund LH } \\
\text { 10. McMurray } \\
\text { JJ }\end{array}$ & 56 & $\begin{array}{l}\text { Nueva York, Estados } \\
\text { Unidos }\end{array}$ \\
\hline
\end{tabular}

publicaciones, fueron Shah SJ, Borlaug BA y Redfield MM, con 105, 99 y 78 artículos publicados, respectivamente; no obstante, en la tabla 2 se incluyen los 10 principales autores y lo concerniente al número de publicaciones y el país de residencia.

En WoS se encontraron 7943 publicaciones teniendo el tema un H-índex de 143, con un promedio de citas

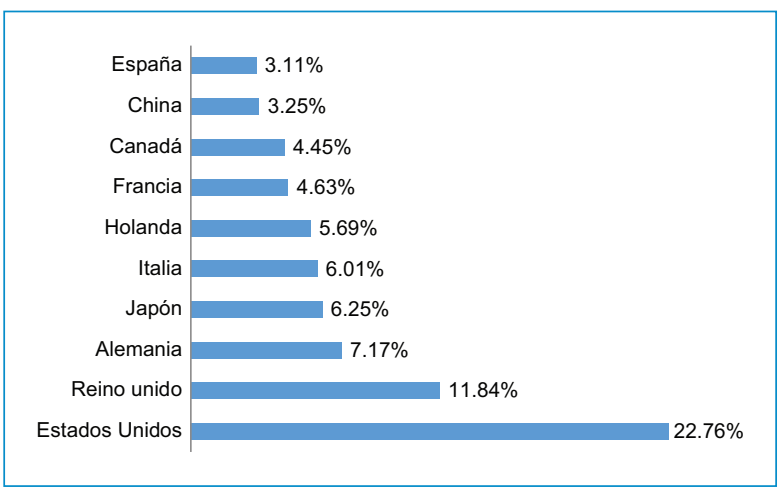

Figura 2. Países con mayor porcentaje de artículos sobre insuficiencia cardiaca con fracción de eyección preservada durante el periodo 1988-2018, según Medline.

de 18.02 y un número total de citaciones de 132,098. Entre los países que más producción científica tuvieron, según WoS, se encuentran los Estados Unidos con 3155 (39.7\%) publicaciones, Japón con 692 (8.7\%) y Alemania con 628 (7.9\%). Las revistas más representativas fueron European Journal of Heart Failure con $720(9.1 \%)$ artículos publicados, Circulation con $597(7.5 \%)$ y Journal of the American College of Cardiology con 411 (5.2\%).

En Scopus se encontraron 4136 publicaciones. Entre los países que más producción científica tuvieron, según Scopus, se encuentran los Estados Unidos con 1840 (44.5\%) publicaciones, el Reino Unido con $431(10.4 \%)$ y Alemania con $353(8.5 \%)$. Las revistas 
más representativas fueron European Journal of Heart Failure con $313(7.6 \%)$ artículos publicados, Journal of the American College of Cardiology con $176(4.2 \%) \mathrm{e}$ International Journal of Cardiology con 172 (4.1\%).

\section{Discusión}

Los datos obtenidos en este análisis bibliométrico, a partir de las bases de datos Medline, Scopus y WoS, indican que es el primer estudio en realizar una recopilación histórica de la producción científica sobre ICFEp, con la intención de señalar el impacto mundial de esta enfermedad y las decisiones en materia de investigación que se están tomando al respecto ${ }^{12,13}$.

En los hallazgos preliminares destaca el volumen literario disponible sobre esta variante de la falla cardiaca, que revela un incremento del $17.36 \%$ en la producción científica durante el periodo de tiempo evaluado (1988-2018); por consiguiente, es un dato que respalda el creciente interés de la comunidad científica por comprender mejor la función cardiaca y por demostrar el compromiso con la población afectada.

El número de publicaciones sobre ICFEp en las bases de datos WoS (7943), Medline (2830) y Scopus (4136) se correlacionan con las características intrínsecas de cada una de estas fuentes literarias. WoS es la base con mayor número de publicaciones, teniendo en cuenta que sus registros e indexaciones se iniciaron en 1900, mientras que Medline data de 1950 y Scopus de 1966; sin embargo, esta última tiene la característica de abarcar más revistas que WoS y Medline ${ }^{14}$.

Estos resultados, no obstante, indican acciones insuficientes en investigación en comparación con otras enfermedades que tienen una mayor densidad de publicaciones ${ }^{15-17,18}$; de hecho, en WoS se encontró que la ICFEp ha presentado cifras notablemente inferiores en cuanto a número total de publicaciones, $\mathrm{H}$-índex por tema y promedio de citas por artículos ${ }^{13,18-20}$, que avalan la problemática en curso, de acuerdo con la alta prevalencia y el déficit de guías para el manejo de esta variante de insuficiencia cardiaca.

En las bases de datos se identificaron las revistas de mayor trascendencia; llama la atención que tanto WoS como Medline y Scopus coincidieron al incluir European Journal of Heart Failure, International Journal of Cardiology y Journal of the American College of Cardiology como las más representativas, resaltando que sus diferencias obedecen a la cantidad de revistas suscritas, así como al tipo de artículos publicados en cada una de ellas.
En el proceso de identificar el papel de las comunidades científicas internacionales, en el contexto de la ICFEp, se evidenció que los países desarrollados son los más involucrados en el campo de la investigación, y que, de acuerdo con la cantidad de publicaciones, son liderados por los Estados Unidos, seguido de Alemania, Japón y el Reino Unido. Así mismo, los autores que más producen en este campo son los de nacionalidad estadounidense, holandesa o alemana, recalcando la imposibilidad de reconocer a los autores adjuntos y secundarios en cada artículo ${ }^{18}$.

Por otro lado, los resultados son desalentadores para los países en desarrollo, y en América Latina el déficit literario sobre la ICFEp es evidente, ya que, pese a tener investigaciones encabezadas por Brasil, este tan solo representa el $0.81 \%$ de las publicaciones totales y Colombia apenas el $0.03 \%$, hecho que hace reflexionar sobre si realmente una patología con tal alta prevalencia y carga de enfermedad merece tan poca relevancia local, considerando que se prevé que será el fenotipo de insuficiencia cardiaca más frecuente ${ }^{19,21}$.

Se debe tener en cuenta que en el proceso de búsqueda en las bases de datos es necesario filtrar la información por artículos escritos en inglés, y por ende existe una alta probabilidad de que haya un sesgo para publicaciones realizadas en otros idiomas y que pudieran tener relevancia para esta investigación. Así mismo, con la posibilidad de tener recursos a la mano, como Science Citation Index, FABUMED y otras bases de datos, se crea la opción de realizar una búsqueda bibliográfica de gran volumen sin dejar atrás la calidad del contenido y la facilidad del acceso.

Un punto relevante derivado del consenso de esta revisión y otras publicaciones mundiales acerca de la ICFEp es la necesidad de modificar los objetivos de los investigadores, ello frente al desconcierto que surge al ser una enfermedad tan compleja y prevalente, con reiterados estudios negativos descritos. Probablemente, las intervenciones deban cambiar y encaminarse hacia la búsqueda de otro tipo de desenlaces, como la mejoría en la calidad de vida y la sintomatología, que al incluirse cada vez más en la ICFEr sin duda generarán cambios significativos para los pacientes ${ }^{22}$.

En la actualidad se evalúan nuevas intervenciones terapéuticas en la ICFEp, lo cual se hace evidente con PARAGON-HF, el más reciente estudio doble ciego y aleatorizado que comparó la seguridad y la eficacia de la nueva molécula sacubitril/valsartán frente a valsartán, cuyos resultados desalentadores no mostraron diferencias estadísticamente significativas en cuanto a la 
reducción de la incidencia de muerte de causa cardiovascular u hospitalización por insuficiencia cardiaca. Sin embargo, se obtuvieron desenlaces secundarios alentadores en cuanto al mejoramiento de la clase funcional, la preservación de la función renal y el aparente beneficio de las mujeres en el desenlace primario del estudio; resultados que evidentemente justifican y motivan la necesidad de continuar investigando nuevas opciones terapéuticas que logren un impacto significativo en el curso de la enfermedad ${ }^{23-25}$.

Similar es el caso del SOCRATES-PRESERVED, un estudio prospectivo, aleatorizado, doble ciego y controlado con placebo, para determinar la tolerancia y la dosis óptima del vericiguat, un estimulador de la guanilato ciclasa, en pacientes con ICFEp crónica, cuyos resultados no mostraron cambios respecto al valor basal de los péptidos natriuréticos ni en el volumen auricular izquierdo a las 12 semanas, pero logró impactar en la calidad de vida de los pacientes con una disminución de síntomas y de limitaciones físicas, lo cual justifica la realización de un estudio adicional ${ }^{24}$.

Además, se evalúa la viabilidad del uso de inhibidores del cotransportador de sodio glucosa tipo 2 (iSGLT2) ${ }^{26,27}$, que al haber obtenido resultados bastante positivos en la ICFEr busca proyectar desenlaces similares en la ICFEp; sin embargo, es un proceso complejo, en el que los investigadores han venido afinando numerosos factores de gran repercusión, como son los criterios de inclusión y exclusión de los estudios y el correcto diagnóstico de la enfermedad, que incluya la clínica, los hallazgos ecocardiográficos y los valores séricos de los péptidos natriuréticos ${ }^{28}$.

Por otro lado, es imprescindible interrogarse acerca del papel de las grandes industrias farmacéuticas, o de los mismos sistemas de salud, si como agentes interventores están dispuestos a invertir en medidas y resultados que no solo tengan impacto en la mortalidad, sino que también empiecen a dar importancia a otros tipos de desenlaces propuestos (calidad de vida, etc.) ${ }^{22}$.

En esta búsqueda bibliométrica sobre ICFEp se encontró que, a pesar del aumento constante de la producción científica en la última década, es imprescindible que todas las comunidades intensifiquen la actitud investigadora, siempre y cuando las grandes industrias farmacéuticas, o los sistemas de salud, estén dispuestos a invertir en medidas diferentes del impacto sobre la mortalidad y empiecen a dar importancia a otro tipo de desenlaces propuestos (calidad de vida, etc.) ${ }^{30}$, reconociendo la trascendencia epidemiológica de esta enfermedad, y con esto se dispongan los recursos necesarios para generar nuevos conocimientos y el consecuente potenciamiento del abordaje integral de la ICFEp ${ }^{26,28-30}$.

\section{Conclusiones}

Este análisis bibliométrico es pionero en señalar la producción, la evolución y la situación de la investigación científica acerca de la ICFEp en los últimos 30 años, evidenciándose una tasa de crecimiento anual del $17.36 \%$ en cuanto al número de publicaciones, lo que representa una asociación directamente proporcional entre la literatura disponible y el nivel de desarrollo de cada país.

En los últimos años, Colombia ha carecido de participación significativa en el desarrollo de este tipo de literatura médica, por lo que se requiere aumentar la cantidad de investigación sobre este tema y lograr una mejor calidad vigilando la rigurosidad metodológica y la finalidad de las intervenciones, con la tentativa de ser pioneros en alternativas de modificación de la sintomatología y la calidad de vida para este complejo tipo de insuficiencia cardiaca.

La realización de este estudio fue motivada por el creciente comportamiento epidemiológico de la ICFEp y la variabilidad de datos en cuanto a mortalidad y supervivencia conforme aumentan la esperanza de vida, la conciencia diagnóstica y la comorbilidad asociada. Estos hallazgos pretenden contextualizar a todas las comunidades e instituciones sanitarias sobre la trascendencia de permanecer en actitud de investigación, teniendo en cuenta que solo así se puede comprender la fisiopatología de la ICFEp y crear guías de manejo adecuadas que permitirán abordar las exigencias clínicas de las diferentes poblaciones y el reto del tratamiento individualizado.

\section{Financiamiento}

Los autores declaran que no recibieron ninguna financiación para la realizar esta publicación.

\section{Conflicto de intereses}

No se declara conflicto de intereses por parte de ninguno de los autores.

\section{Responsabilidades éticas}

Protección de personas y animales. Los autores declaran que para esta investigación no se han realizado experimentos en seres humanos ni en animales. 
Confidencialidad de los datos. Los autores declaran que en este artículo no aparecen datos de pacientes.

Derecho a la privacidad y consentimiento informado. Los autores declaran que en este artículo no aparecen datos de pacientes.

\section{Bibliografía}

1. Derrick G, Jonkers K, Lewison G. Characteristics of bibliometrics articles in library and information sciences (LIS) and other journals. En: Proceedings, $17^{\text {th }}$ International Conference on Science and Technology Indicators. Montreal, Canada; 2012. p. 449-551.

2. Gómez E. Capítulo 2. Introducción, epidemiología de la falla cardiaca e historia de las clínicas de falla cardiaca en Colombia. Rev Colomb Cardiol. 2016;23:6-12.

3. Ganpule AP, Vijayakumar M, Malpani A, Desai MR. Percutaneous nephrolithotomy (PCNL), a critical review. Int J Surg. 2016:36:660-4.

4. Ponikowski P, Voors AA, Anker SD, Bueno H, Cleland JG, Coats AJ, et al. 2016 ESC Guidelines for the diagnosis and treatment of acute and chronic heart failure: The Task Force for the diagnosis and treatment of acute and chronic heart failure of the European Society of Cardiology (ESC). Developed with the special contribution of the Heart Failure Association (HFA) of the ESC. Eur J Heart Fail. 2016;18:891-975.

5. Alonso Betanzos A, Bolón Canedo V, Heyndrickx GR, Kerkhof PL. Exploring guidelines for classification of major heart failure subtypes by using machine learning. Clin Med Insights Cardiol. 2015;9(Suppl 1):57-71.

6. Metra M, Teerlink JR. Heart failure. Lancet. 2017;390:1981-95.

7. Gurwitz JH, Magid DJ, Smith DH, Goldberg RJ, McManus DD, Allen LA, et al. Contemporary prevalence and correlates of incident heart failure with preserved ejection fraction. Am J Med. 2013;126:393-400.

8. Yancy CW, Jessup M, Bozkurt B, Butler J, Casey DE, Drazner MH, et al. 2013 ACCF/AHA guideline for the management of heart failure: a report of the American College of Cardiology Foundation/American Heart Association Task Force on Practice Guidelines. J Am Coll Cardiol. 2013;62:e147-e239.

9. Mozaffarian D, Benjamin EJ, Go AS, Arnett DK, Blaha MJ, Cushman M, et al. Heart disease and stroke statistics - 2016 update: a report from the American Heart Association. Circulation. 2016;133:e38-e360.

10. Iliesiu AM, Hodorogea AS. Treatment of heart failure with preserved ejection fraction. Adv Exp Med Biol. 2018;1067:67-87.

11. Rodríguez-Morales AJ. La importancia del $\mathrm{H}$ índex como indicador de la producción y la calidad científica. Sci Tech. 2015;20(3).

12. Khan MS, Ullah W, Riaz IB, Bhulani N, Manning WJ, Tridandapani S, et al. Top 100 cited articles in cardiovascular magnetic resonance: a bibliometric analysis. J Cardiovasc Magn Reson. 2017:18:87.

13. Ugolini D, Neri M, Cesario A, Marazzi G, Milazzo D, Volterrani M, et al. Bibliometric analysis of literature in cerebrovascular and cardiovascular diseases rehabilitation: growing numbers, reducing impact factor. Arch Phys Med Rehabil. 2013;94:324-31.
14. Shuaib W, Khan MS, Shahid H, Valdes EA, Alweis R. Bibliometric analysis of the top 100 cited cardiovascular articles. Am J Cardiol. 2015;115:972-81.

15. Michalopoulos A, Falagas ME. A bibliometric analysis of global research production in respiratory medicine. Chest. 2005:128:3993-8.

16. Sweileh WM, Sa'ed HZ, Al-Jabi SW, Sawalha AF. Bibliometric analysis of diabetes mellitus research output from Middle Eastern Arab countries during the period (1996-2012). Scientometrics. 2014:101:819-32.

17. Falagas ME, Pitsouni El, Malietzis GA, Pappas G. Comparison of PubMed, Scopus, Web of Science, and Google Scholar: strengths and weaknesses. FASEB J. 2008;22:338-42.

18. Tian J, Li M, Lian F, Tong $X$. The hundred most-cited publications in microbiota of diabetes research: a bibliometric analysis. Medicine (Baltimore). 2017;96:e7338.

19. Seriwala HM, Khan MS, Shuaib W, Shah SR. Bibliometric analysis of the top 50 cited respiratory articles. Expert Rev Respir Med. 2015;9:817-24.

20. Lam CS, Lyass A, Kraigher-Krainer E, Massaro JM, Lee DS, Ho JE, et al. Cardiac dysfunction and noncardiac dysfunction as precursors of heart failure with reduced and preserved ejection fraction in the community. Circulation. 2011;124:24-30.

21. Zakeri R, Cowie MR. Heart failure with preserved ejection fraction: controversies, challenges and future directions. Heart. 2018;104:377-84.

22. Boehringer Ingelheim. EMPagliflozin outcomE tRial in Patients With chrOnic heaRt Failure With Preserved Ejection Fraction (EMPEROR-Preserved) - Tabular View. ClinicalTrials.gov. 2017. Disponible en: https:// clinicaltrials.gov/ct2/show/record/NCT03057951.

23. Solomon SD, McMurray JJV, Anand IS, Ge J, Lam CSP, Magggioi AP, et al. Angiotensin-neprilysin inhibition in heart failure with preserved ejection fraction. N Engl J Med. 2019;381:1609-20.

24. Solomon S, Rizkala A, Gong J, Wang W, Ananda IS, Ge J, et al. Angiotensin receptor neprilysin inhibition in heart failure with preserved ejection fraction: rationale and design of the PARAGON-HF Trial. JACC Heart Fail. 2017;5:471-82

25. Ndumele C, Matsushita K, Sang Y, Lazo M, Agarwal K, Nambi V, et al. $\mathrm{N}$-terminal pro-brain natriuretic peptide and heart failure risk among individuals with and without obesity: the Atherosclerosis Risk in Communities (ARIC) Study. Circulation. 2016;133:631-8.

26. Hogg K, Swedberg K, McMurray J. Heart failure with preserved left ventricular systolic function: epidemiology, clinical characteristics, and prognosis. Am Coll Cardiol. 2004;43:317-27.

27. Tang X, Gong W, Yuan F, Li R, Han X, Huang S, et al. Top-cited articles in digestive system disease from 1950 to 2013. J Gastroenterol Hepatol. 2016;31:107-11.

28. Parikh KS, Sharma K, Fiuzat M, Surks HK, George JT, Honarpour N, et al. Heart failure with preserved ejection fraction expert panel report: current controversies and implications for clinical trials. JACC Heart Fail. 2018;6:619-32

29. Nishimura RA, Otto CM, Bonow RO, Carabello BA, Erwin JP, Fleisher LA, et al. AHA/ACC Focused Update of the 2014 AHA/ACC Guideline for the management of patients with valvular heart disease: a report of the American College of Cardiology/American Heart Association Task Force on Clinical Practice Guidelines. J Am Coll Cardiol. 2017;70:252-89.

30. Pieske B, Maggioni AP, Lam CSP, Pieske-Kraigher E, Filippatos G, Butler $\mathrm{J}$, et al. Vericiguat in patients with worsening chronic heart failure and preserved ejection fraction: results of the SOluble guanylate Cyclase stimulatoR in heArT failurE patientS with PRESERVED EF (SOCRATES-PRESERVED) study. Eur Heart J. 2017;38:1119-27. 